\title{
NÍVEIS CRÍTICOS DE FÓSFORO, PARA EUCALIPTO, EM CASA DE VEGETAÇÃO, EM FUNÇÃO DA SUA LOCALIZAÇÃO NO SOLO(1)
}

\author{
Paulo Batista Leite ${ }^{(2)}$, Víctor Hugo Alvarez V. ${ }^{(3)}$, Nairam Félix de \\ Barros $^{(3)}$, Júlio César Lima Neves ${ }^{(3)}$, André Guarçoni M. ${ }^{(4)} \&$ Luiz \\ Antônio Zanão Júnior ${ }^{(5)}$
}

\begin{abstract}
RESUMO
A disponibilidade do $\mathrm{P}$ é modificada pela aplicação localizada desse elemento no solo, pois diminui sua adsorção, precipitação e fixação, pela redução do contato com o solo. Estudou-se, em casa de vegetação, a calibração de $P$ em função da diferença de teores de $\mathbf{P}$ do solo provocada por sua aplicação localizada. Foram utilizadas amostras de Latossolos Vermelho-Amarelos distróficos com textura muito argilosa e de outro com textura média. Utilizaram-se 14 combinações de sete doses de $\mathbf{P}$ adicionadas nas partes central e superior da amostra do vaso ("cova") com outras sete doses de $P$ adicionadas no solo circulante à "cova" ("solo externo"). As amostras dos dois locais foram incubadas por 40 dias com $P$ em embalagens separadas e depois acondicionadas no vaso. Uma muda de Eucalyptus pellita foi cultivada na parte média da "cova" de cada vaso por 82 dias. Determinaram-se a produção de matéria seca da parte aérea (MS) e o teor de $P$ por Mehlich-1 nos dois locais do solo em amostras colhidas depois da incubação e antes do plantio e em uma amostra composta pelo solo da "cova" mais o "externo" da parte superior, após a colheita. O efeito da localização foi evidente, pois a produção de MS não se relacionou bem com a quantidade de $P$ aplicada por vaso, e sim com as doses aplicadas nos dois locais. $O$ crescimento da planta dependeu essencialmente da dose aplicada na "cova". Valores elevados do nível crítico para a "cova" não implicam uso de grande quantidade de $P$ por vaso. $O$ teor de $P$ na amostra após a colheita
\end{abstract}

\footnotetext{
(1) Parte da Tese de Mestrado do primeiro autor apresentada ao curso de Solos e Nutrição de Plantas da Universidade Federal de Viçosa - UFV. Recebido para publicação em setembro de 2008 e aprovado em julho de 2009.

(2) Engenheiro-Agrônomo, M.Sc. Pioneer Sementes Ltda. CEP 75500-000 Itumbiara (GO). E-mail: paulo.leite@pioneer.com

(3) Professor do Departamento de Solos, Universidade Federal de Viçosa - UFV. CEP 36571-000 Viçosa (MG). Bolsista do CNPq. E-mails: vhav@ufv.br; nfbarros@ufv.br; julio_n2003@yahoo.com.br

(4) Pesquisador do Instituto Capixaba de Pesquisa, Assistência Técnica e Extensão Rural - INCAPER, CEP 29375-000 Venda Nova do Imigrante (ES). E-mail: guarconi@incaper.es.gov.br

(5) Pesquisador do Instituto Agronômico do Paraná - IAPAR. Unidade Regional de Pesquisa do Centro Sul do Paraná, CEP 84001970 Ponta Grossa (PR). E-mail: lzanao@iapar.br
} 
representou bem a disponibilidade média, porém não se relacionou bem com a produção de MS, o que dificulta a obtenção de um nível crítico para amostras que representem a fertilidade média para todo o volume de solo. Mudanças no método de calibração de $P$ são sugeridas em condições de aplicação localizada do $P$.

Termos de indexação: amostragem, $P$ disponível, calibração, doses recomendáveis.

\title{
SUMMARY: CRITICAL PHOSPHORUS LEVELS FOR GREENHOUSE- GROWN EUCALYPTUS AS RELATED TO THE PLACEMENT OF FERTILIZER P IN THE SOIL
}

\begin{abstract}
The availability of $P$ is influenced by the application location of this element to the soil, affecting the adsorption, precipitation and fixation due to the reduction of contact with the soil. This study, carried out in a greenhouse, aimed to calibrate P soil concentrations for Eucalyptus pellita growth as affected by fertilizer placement. Samples of two Oxisols were used. The treatment consisted of 14 combinations of seven P rates, which were placed in holes in the upper mid part of pots (planting hole), and seven $P$ doses applied to the soil around the holes (external soil). Samples of the two soils were incubated separately with $P$ for 40 days before being placed in the pots. One eucalyptus plant was grown in the middle of the fertilizer hole of each pot for 82 days. The shoot dry matter was determined and soil samples were collected from both positions of fertilizer placement before sowing and after harvesting the plant, plus a composite sample of both soil positions after harvest. The soil P content was determined by Mehlich-1. Dry matter yield was not closely related with the applied $P$ per pot, but was related with the rates applied in both positions, which demonstrated the effect of location. Plant growth depended essentially on the dose applied in the "hole". The high critical P level determined for this placement does not express the need of using high amounts of P per pot. The P content determined in the upper soil part after harvest clearly represented the mean P availability, but was not closely related with plant growth, indicating the difficulty of establishing a critical level that would represent the mean fertility of the whole soil volume. We suggest adjustments in the calibration method for P when the element is locally applied.
\end{abstract}

Index terms: sampling, available $P$, calibration, recommended doses.

\section{INTRODUÇÃO}

A maioria dos solos brasileiros caracteriza-se pela elevada capacidade de adsorção de $\mathrm{P}$ e por apresentar baixos teores desse elemento em solução, o que torna necessária a prática de localizar fertilizantes fosfatados solúveis em parte do volume de solo cultivado, no sentido de reduzir a fixação do $\mathrm{P}$ e otimizar a sua absorção pelas plantas.

A aplicação do P em fração do volume de solo tem a vantagem de protegê-lo contra as reações de adsorção, precipitação e de fixação, uma vez que diminui seu contato com o solo. Com isso, na fração fertilizada com fonte fosfatada solúvel haverá alto teor do nutriente em forma disponível (Model \& Anghinoni, 1992) e, na fração restante, ele permanecerá inalterado, uma vez que o P é praticamente imóvel no solo (Bray, 1954). Assim, quando se aplica uma fonte fosfatada no sulco de plantio ou na cova, a planta inicialmente explora no solo uma região com $\mathrm{P}$ em maior disponibilidade e, à medida que cresce, passa a explorar um volume de solo com menor disponibilidade. Esse fato para o eucalipto torna-se muito importante, pois sabe-se que o nível crítico de $\mathrm{P}$ no solo é maior quanto mais jovens são as plantas e decrescem com a idade destas (Novais et al., 1982). Esses autores estimaram que o nível crítico de $\mathrm{P}$ no solo era de $52 \mathrm{mg} \mathrm{dm}^{-3}$ aos 85 dias e caiu para $12 \mathrm{mg} \mathrm{dm}^{-3}$ aos 133 dias de idade. Atribuiuse esse decréscimo às variações no crescimento radicular e, ou, a possíveis alterações metabólicas das plantas.

Para uma prática racional de adubação, otimizando recursos econômicos e ambientais, é necessário um criterioso estudo de calibração. O nível crítico nesses estudos é definido como a concentração do nutriente no solo que corresponde à disponibilidade necessária para atingir a produção de máxima eficiência econômica (Alvarez V., 1996). No entanto, nos trabalhos de calibração para $\mathrm{P}$, a forma de localização não tem sido considerada, e o que se faz é relacionar a resposta da cultura à disponibilidade obtida com a aplicação do nutriente a todo volume de solo para contemplar as exigências das culturas. Assim, tornase difícil definir a disponibilidade do nutriente para as plantas pelo método tradicional, com definição de 
um valor de nível crítico indiferente à localização do fertilizante, já que, com a localização, a resposta da planta passará a ser função das diferentes concentrações de $\mathrm{P}$ no volume de solo explorado pelas raízes. Os trabalhos de calibração de $\mathrm{P}$ devem considerar as condições de aplicação dos fertilizantes e a forma de amostragem dos solos para as quais as recomendações serão geradas, ou seja, a forma como serão utilizados pelos agricultores.

Vários autores verificaram que a relação entre as quantidades de $\mathrm{P}$ recuperadas do solo com as doses adicionadas varia com a localização do adubo e, consequentemente, com a forma de amostragem (Vasconcelos et al., 1982; Alvarez V., 1996; Guarçoni M., 2001). Logo, os níveis críticos variam do mesmo modo. Assim, quando se faz a aplicação localizada do $\mathrm{P}$, o modo de amostragem do solo para análise tornase muito importante, pois o resultado desta não deve expressar apenas a disponibilidade do nutriente representativa do volume de solo, mas também aquela que se relacione com a resposta da cultura.

Dessa forma, em face de uma situação em que a localização do fertilizante venha a definir duas regiões com diferentes teores de $\mathrm{P}$, o modelo de calibração deverá ser ajustado na sua forma de definição de níveis críticos. Esses ajustes deverão incluir a padronização da forma de amostragem do solo.

Este trabalho objetivou estudar a calibração de $\mathrm{P}$ disponível (extrator Mehlich-1) para o crescimento de plantas de eucalipto em função da diferença de teores no solo, provocada por sua aplicação localizada.

\section{MATERIAL E MÉTODOS}

No experimento, realizado em casa de vegetação, foi utilizada amostra de um Latossolo VermelhoAmarelo distrófico textura muito argilosa coletada na camada de 20-50 cm de profundidade, em Viçosa-MG (solo LVAd-VI), e de um Latossolo Vermelho-Amarelo distrófico textura média, coletada nos $20 \mathrm{~cm}$ superficiais, em Lassance-MG (solo LVAd-LS) (Quadro 1).

Os tratamentos derivaram da combinação de sete doses de $\mathrm{P}$ aplicadas em $0,53 \mathrm{dm}^{3}$ de solo, equivalentes a $10 \%$ do volume total, na parte central e superior da amostra do vaso ("cova"), com sete doses de P nos $4,77 \mathrm{dm}^{3}$ de solo circundante ("externo") (Figura 1). Foram utilizadas as 13 combinações da matriz experimental "Box - Berard aumentada (3)" (Leite, 1984), mais dois tratamentos adicionais: um, em cuja combinação a dose de $\mathrm{P}$ da "cova" foi menor que a dose do solo "externo" (T14), e outro, em que a combinação correspondente ao ponto central da matriz utilizada $(0,00: 0,00)$ foi repetida, porém sem incubação e com o P do solo "externo" localizado (T15) (Figura 1 e Quadro 2). Os 15 tratamentos foram distribuídos em blocos casualizados com cinco repetições.
Quadro 1. Características químicas e físicas das amostras de solos utilizadas no experimento

\begin{tabular}{lcc}
\hline \multirow{2}{*}{ Característica } & \multicolumn{2}{c}{ Solo } \\
\cline { 2 - 3 } & LVAd-VI ${ }^{(1)}$ & LVAd-LS $^{(1)}$ \\
\hline $\mathrm{pH}\left(\mathrm{H}_{2} \mathrm{O}, 1: 25\right)$ & 4,40 & 4,70 \\
$\mathrm{Al}^{3+}\left(\mathrm{cmol}_{\mathrm{c}} \mathrm{dm}^{-3}\right)^{(2)}$ & 1,00 & 0,60 \\
$\mathrm{Ca}^{2+}\left(\mathrm{cmol}_{\mathrm{c}} \mathrm{dm}^{-3}\right)^{(2)}$ & 0,10 & 0,30 \\
$\mathrm{Mg}^{2+}\left(\mathrm{cmol}_{\mathrm{c}} \mathrm{dm}^{-3}\right)^{(2)}$ & 0,04 & 0,03 \\
$\mathrm{H}+\mathrm{Al}_{\left(\mathrm{cmol}_{\mathrm{c}} \mathrm{dm}^{-3}\right)^{(3)}}$ & 5,70 & 3,60 \\
$\mathrm{P}\left(\mathrm{mg} \mathrm{dm}^{-3}\right)^{(4)}$ & 1,00 & 0,70 \\
$\mathrm{~K}\left(\mathrm{mg} \mathrm{dm}^{-3}\right)^{(4)}$ & 9,00 & 36,00 \\
$\mathrm{P} \mathrm{rem}\left(\mathrm{mg} \mathrm{L}^{-1}\right)^{(5)}$ & 4,00 & 16,00 \\
$\mathrm{CO}\left(\mathrm{g} \mathrm{kg}^{-1}\right)$ & 20,0 & 11,0 \\
$\mathrm{Capacidade} \mathrm{de} \mathrm{campo}\left(\mathrm{kg} \mathrm{kg}^{-1}\right)$ & 0,32 & 0,22 \\
Areia grossa (\%) & 13,00 & 44,00 \\
Areia fina (\%) & 16,00 & 17,00 \\
Silte (\%) & 5,00 & 6,00 \\
Argila (\%) & 66,00 & 33,00
\end{tabular}

Classificação textural Muito argiloso Franco-argilosoarenoso

(1) LVAd-VI: Latossolo Vermelho-Amarelo distrófico, do Tiro de Guerra de Viçosa-MG; LVAd-LS: Latossolo Vermelho-Amarelo, de Lassance-MG. ${ }^{(2)}$ Extrator $\mathrm{KCl} 1 \mathrm{~mol} \mathrm{~L}^{-1} .{ }^{(3)}$ Extrator $\mathrm{Ca}(\mathrm{OAc})_{2} 0,5 \mathrm{~mol} \mathrm{~L}^{-1}$, pH 7. ${ }^{(4)}$ Extrator Mehlich-1. ${ }^{(5)} \mathrm{P}$ rem: fósforo remanescente (Alvarez V. et al., 2000).

Não foi realizada calagem, razão pela qual a dose de $\mathrm{Ca}$ foi equilibrada por meio das fontes de $\mathrm{P}$, enquanto o $\mathrm{Mg}$ foi aplicado na forma de $\mathrm{MgCO}_{3}$. As doses de N, S e K foram iguais no solo da "cova" e no solo "externo". Os micronutrientes foram fornecidos durante o ensaio, em forma de solução.

As doses de $\mathrm{P}$ (Quadro 2), nos dois solos, foram escolhidas dentro de diferentes espaços experimentais, por apresentarem diferentes capacidades-tampão (Alvarez V. \& Fonseca, 1990). As doses usadas no solo LVAd-LS correspondem às utilizadas no solo LVAdVI divididas por 1,75. As quantidades totais de $\mathrm{P}$ aplicadas por vaso variaram de acordo com a combinação de doses. Foram utilizados, como fontes de $\mathrm{P}$, o superfosfato simples, o superfosfato triplo, $\mathrm{KH}_{2} \mathrm{PO} 4$ e $\mathrm{NH}_{4} \mathrm{H}_{2} \mathrm{PO}_{4}$, em quantidades limitadas pelas doses dos nutrientes acompanhantes.

As amostras de solo que receberam as doses de $\mathrm{P}$, e os demais nutrientes foram incubados por 40 dias. Ao final da incubação e após a retirada de amostras para determinação de P, os solos da "cova" e "solo externo" foram acondicionados nos vasos (Figura 1). Cada vaso recebeu, então, duas mudas de Eucalyptus pellita, crescidas em canteiros de areia esterilizada, que foram transplantadas na parte média do vaso "cova", quando tinham em média seis pares de folhas. Quinze dias após o transplantio, foi efetuado desbaste, deixando-se a planta de melhor aspecto vegetativo em cada vaso. Após 82 dias do desbaste, fez-se o corte da 
Quadro 2. Doses de P para a "cova" (C) e para o solo "externo" (E) e quantidades de P aplicadas por vaso, nas amostras dos solos LVAd-VI e LVAd-LS

\begin{tabular}{|c|c|c|c|c|c|}
\hline \multicolumn{2}{|c|}{ Tratamento } & \multicolumn{2}{|c|}{ LVAd-VI } & \multicolumn{2}{|c|}{ LVAd-LS ${ }^{(3)}$} \\
\hline $\mathrm{N}^{\circ}$ & $\mathbf{C}+\mathbf{E}$ & $\begin{array}{c}\text { Dose de } P \\
C+E\end{array}$ & $\begin{array}{c}\text { P aplicado por } \\
\text { vaso }\end{array}$ & $\begin{array}{c}\text { Dose de P } \\
C+E\end{array}$ & $\begin{array}{c}\mathrm{P} \text { aplicado por } \\
\text { vaso }\end{array}$ \\
\hline & Níveis & $\mathrm{mg} \mathrm{dm} \mathrm{m}^{-3}$ & $\mathrm{mg} / \mathrm{vaso}$ & $\mathrm{mg} \mathrm{dm}{ }^{-3}$ & $\mathrm{mg} / \mathrm{vaso}$ \\
\hline $\mathrm{T} 1$ & $-0,50:-0,50$ & $175,0+35,0$ & 259,7 & $100,0+20,0$ & 148,4 \\
\hline $\mathrm{T} 2$ & $-0,50: 0,50$ & $175,0+105,0$ & 593,6 & $100,0+60,0$ & 339,2 \\
\hline $\mathrm{T} 3$ & $0,50:-0,50$ & $525,0+35,0$ & 445,2 & $300,0+20,0$ & 254,4 \\
\hline $\mathrm{T} 4$ & $0,50: 0,50$ & $525,0+105,0$ & 779,1 & $300,0+60,0$ & 445,2 \\
\hline $\mathrm{T} 5$ & $0,00: 0,00$ & $350,0+70,0$ & 519,4 & $200,0+40,0$ & 296,8 \\
\hline $\mathrm{T} 6$ & $-0,75: 0,00$ & $87,5+70,0$ & 380,3 & $50,0+40,0$ & 217,3 \\
\hline $\mathrm{T} 7$ & $0,75: 0,00$ & $612,5+70,0$ & 658,5 & $350,0+40,0$ & 376,3 \\
\hline $\mathrm{T} 8$ & $0,00:-0,75$ & $350,0+17,5$ & 269,0 & $200,0+10,0$ & 153,7 \\
\hline Т 9 & $0,00: 0,75$ & $350,0+122,5$ & 769,8 & $200,0+70,0$ & 439,9 \\
\hline $\mathrm{T} 10$ & $-0,90:-0,50$ & $35,0+35,0$ & 185,5 & $20,0+20,0$ & 106,0 \\
\hline $\mathrm{T} 11$ & $-0,50:-0,90$ & $175,0+7,0$ & 126,1 & $100,0+4,0$ & 72,1 \\
\hline $\mathrm{T} 12$ & $0,90: 0,50$ & $665,0+105,0$ & 853,3 & $380,0+60,0$ & 487,6 \\
\hline $\mathrm{T} 13$ & $0,50: 0,90$ & $525,0+133,0$ & 912,7 & $300,0+76,0$ & 521,5 \\
\hline $\mathrm{T} 14$ & $-0,90: 0,90^{(1)}$ & $35,0+133,0$ & 653,0 & $20,0+76,0$ & 373,1 \\
\hline $\mathrm{T} 15$ & $0,00: 0,00 \mathrm{~L}^{(2)}$ & $350,0+70,0 \mathrm{~L}$ & 519,4 & $200,0+40,0$ & 296,8 \\
\hline
\end{tabular}

${ }^{(1)}$ Tratamento adicional à matriz Box-Berard aumentada (3) (Leite, 1984). ${ }^{(2)}$ Tratamento equivalente ao T5, mas onde não houve incubação antes do plantio e a dose de P do solo "externo" foi localizada (L). ${ }^{(3)}$ As doses usadas no solo LVAd-LS correspondem às utilizadas no solo LVAd-VI divididas por 1,75 .

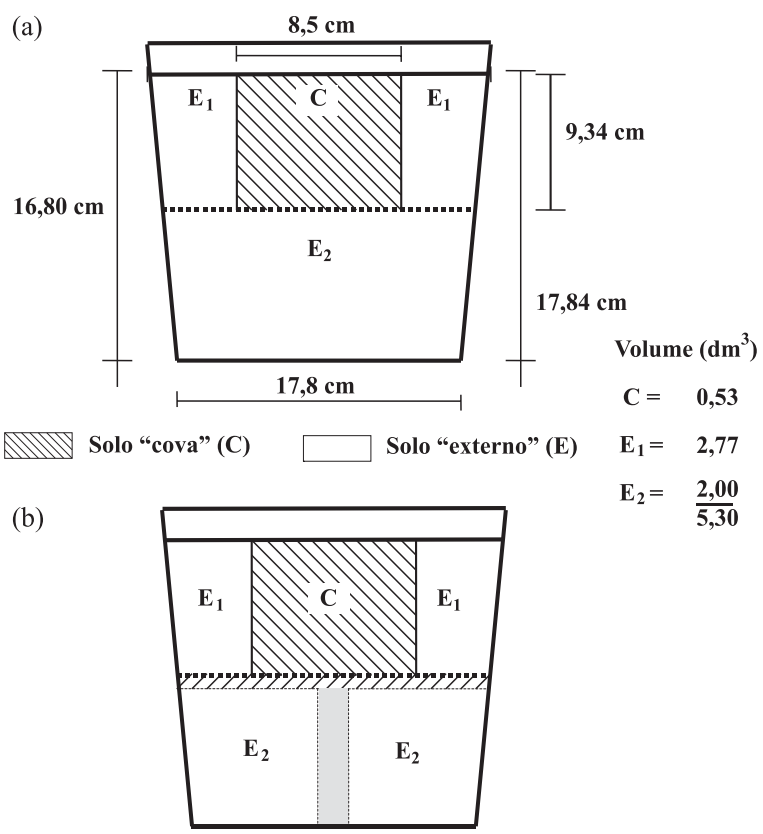

Solo "cova" (C) VIIIIIA Localização $\mathrm{PE}_{1}$ (Superfosfato simples) Solo "externo" (E) $\square$ Localização $\mathrm{PE}_{2}$ (Superfosfato triplo)

Figura 1. Localização do $P$ (a) nos 14 primeiros tratamentos e (b) no $15^{\circ}$ tratamento.

parte aérea, que foi seca em estufa de ventilação forçada, a $70^{\circ} \mathrm{C}$, para determinação da produção de matéria seca (MS). Em duas repetições, retirou-se uma amostra representativa da parte superior do vaso, incluindo "cova" e "solo externo" $\left(\mathrm{C}+\mathrm{E}_{1}\right.$, Figura 1), para determinação de $\mathrm{P}$ disponível.

Considerou-se, como índice de eficiência do $\mathrm{P}$ adicionado ao vaso, a quantidade de matéria seca da parte aérea produzida por unidade de $\mathrm{P}$ adicionada (kg de MS por kg de $\mathrm{P}$ aplicado).

As equações de regressão foram selecionadas após a análise de variância, testando-se os coeficientes dos componentes de cada modelo até $10 \%$. Foram escolhidos os modelos significativos com maior coeficiente de determinação $\left(\mathrm{R}^{2}\right)$.

\section{RESULTADOS E DISCUSSÃO}

\section{Fósforo disponível no solo e produção de matéria seca da parte aérea}

Apesar de as doses aplicadas ao solo LVAd-VI terem sido maiores, os teores de $\mathrm{P}$ nos dois solos foram semelhantes, refletindo o maior desgaste do extrator Mehlich-1 no solo de maior poder-tampão (LVAd-VI muito argiloso), em relação ao solo LVAd-LS (textura média), como discutido por Holford (1997) e Novais \& Smyth (1999) (Quadro 3).

Os teores de P na "cova" (Pc) e no "solo externo" $(\mathrm{Pe})$ não guardaram relação direta com a quantidade total de $\mathrm{P}$ adicionada por vaso e sim com as doses 
Quadro 3. Teores de $\mathrm{P}$ disponível pelo extrator Mehlich-1 após incubação dos solos com as doses de $\mathrm{P}$ aplicadas na "cova" (Pc) e na parte "externa" (Pe)

\begin{tabular}{|c|c|c|c|c|c|}
\hline \multicolumn{2}{|c|}{ Tratamento } & \multicolumn{2}{|c|}{ LVAd-VI } & \multicolumn{2}{|c|}{ LVAd-LS } \\
\hline $\mathrm{N}^{\circ}$ & $\mathbf{C}+\mathbf{E}$ & Cova & Externo & Cova & Externo \\
\hline & Níveis & & 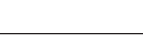 & & \\
\hline $\mathrm{T} 1$ & $-0,50:-0,50$ & 23,00 & 3,42 & 24,21 & 4,31 \\
\hline $\mathrm{T} 2$ & $-0,50: 0,50$ & 24,36 & 12,43 & 24,67 & 12,97 \\
\hline $\mathrm{T} 3$ & $0,50:-0,50$ & 98,50 & 3,42 & 93,32 & 4,88 \\
\hline $\mathrm{T} 4$ & $0,50: 0,50$ & 103,10 & 12,61 & 95,68 & 12,86 \\
\hline $\mathrm{T} 5$ & $0,00: 0,00$ & 64,33 & 7,66 & 62,19 & 9,08 \\
\hline $\mathrm{T} 6$ & $-0,75: 0,00$ & 10,22 & 8,09 & 10,13 & 9,44 \\
\hline $\mathrm{T} 7$ & $0,75: 0,00$ & 120,15 & 8,06 & 108,05 & 10,08 \\
\hline $\mathrm{T} 8$ & $0,00:-0,75$ & 55,61 & 1,68 & 60,93 & 3,30 \\
\hline T 9 & $0,00: 0,75$ & 57,48 & 15,43 & 57,63 & 15,81 \\
\hline $\mathrm{T} 10$ & $-0,90:-0,50$ & 3,62 & 3,70 & 4,35 & 4,38 \\
\hline $\mathrm{T} 11$ & $-0,50:-0,90$ & 21,61 & 0,79 & 24,49 & 1,60 \\
\hline $\mathrm{T} 12$ & $0,90: 0,50$ & 136,36 & 12,84 & 125,99 & 14,37 \\
\hline $\mathrm{T} 13$ & $0,50: 0,90$ & 100,39 & 17,27 & 95,89 & 18,70 \\
\hline $\mathrm{T} 14$ & $-0,90: 0,90$ & 3,08 & 18,28 & 4,14 & 17,45 \\
\hline $\mathrm{T} 15$ & $0,00: 0,00 L^{(1)}$ & - & & - & - \\
\hline
\end{tabular}

(1) Tratamento adicional em que não houve incubação e a dose de Pe foi aplicada de forma localizada.

(Quadro 3). As equações de regressão para os teores de $\mathrm{P}$ recuperados da "cova" e do "solo externo", em função das doses aplicadas às respectivas regiões, apresentaram excelente grau de ajuste (Quadro 4).

A produção de matéria seca (MS) da parte aérea das plantas de eucalipto foi altamente influenciada pela forma de localização do fósforo no solo (Quadro 5), como mostrado pela estreita relação entre massa de MS e as doses de P aplicadas à "cova" e ao "solo externo" (Quadro 6).

Por outro lado, a produção de MS apresentou relação muito baixa com a quantidade total de $\mathrm{P}$ aplicada ao vaso (Quadro 6). No tratamento adicional, a localização da dose de Pe proporcionou produção, nos dois solos, duas vezes superior à do tratamento em que a mesma dose de $\mathrm{P}$ foi misturada a todo $\mathrm{O}$ volume de "solo externo". Neves et al. (1987) verificaram que, para menores quantidades de $\mathrm{P}$, a aplicação localizada proporcionou maior crescimento de mudas de eucalipto e que o aumento nas doses de $\mathrm{P}$ na "cova" incrementou a produção de raízes.

A grande diferença, dentro de cada solo, entre os valores do coeficiente de utilização do $\mathrm{P}$ adicionado reforça o efeito da localização do P (Quadro 5). Esse coeficiente foi maior para aqueles tratamentos que receberam maiores doses de P na "cova" e, ou, menores doses no "solo externo".

Para explicitar o efeito da localização do $\mathrm{P}$, são comparadas as respostas dos tratamentos T14 $\left(35+133 \mathrm{mg} \mathrm{dm}^{-3}\right), \mathrm{T} 5(350+70), \mathrm{T} 3(525+35)$ e T15 $\left(350+70 \mathrm{~L} \mathrm{mg} \mathrm{dm}^{-3}\right)$ no LVAd-VI. O tratamento adicional T14 (35 + $133 \mathrm{mg} \mathrm{dm}^{-3}$ na "cova" e no solo "externo") apresentou produção de MS da parte aérea das plantas de eucalipto de 0,37 g/vaso e eficiência de utilização do $\mathrm{P}$ adicionado de $0,6 \mathrm{~kg}$ de $\mathrm{MS}$ por $\mathrm{kg}$ de $\mathrm{P}$, com adição de $653 \mathrm{mg} /$ vaso de $\mathrm{P}$. A produção de MS do tratamento T5 (350-70) foi de 9,43 g/vaso, ou seja, $2448 \%$ a mais do que a do T14, com uso de menor quantidade de $\mathrm{P}(519,4 \mathrm{mg} / \mathrm{vaso})$, tratamento que também aumentou a eficiência do $\mathrm{P}$ adicionado,

Quadro 4. Equações de regressão para os teores de $P$ recuperado ( $\mathrm{Pr}, \mathrm{em} \mathrm{mg} \mathrm{dm}^{-3}$ de $\mathrm{P}$ ) pelo extrator Mehlich-1 do solo da "cova" e do solo "externo", após incubação e antes do plantio do eucalipto em função das doses de $\mathrm{P}\left(\mathrm{mg} \mathrm{dm} \mathrm{dm}^{-3} \mathrm{de} \mathrm{P}\right)$, aplicadas na "cova" (Pc) ou no solo "externo" (Pe), nos solos LVAd-VI e LVAd-LS

\begin{tabular}{|c|c|c|}
\hline Localização & Equação & $\mathbf{R}^{2}$ \\
\hline \multicolumn{3}{|c|}{ Solo LVAd-VI } \\
\hline Cova & $\hat{\mathrm{P}}_{\mathrm{r}}=-2,735+0,13706^{* *} \mathrm{Pc}+0,000110^{* *} \mathrm{Pc}^{2}$ & 0,997 \\
\hline "Externo" $\hat{\mathrm{P}}$ & $\hat{\mathrm{P}}_{\mathrm{r}}=0,223+0,08241^{* *} \mathrm{Pe}+0,000360^{*} \mathrm{Pe}^{2}$ & 0,997 \\
\hline \multicolumn{3}{|c|}{ Solo LVAd-LS } \\
\hline Cova & $\hat{\mathrm{P}}_{\mathrm{r}}=-3,178+0,28216^{* *} \mathrm{Pc}+0,000140^{*} \mathrm{Pc}^{2}$ & 0,997 \\
\hline "Externo" $\hat{P}$ & $\hat{\mathrm{P}}_{\mathrm{r}}=-0,391+0,22441{ }^{* *} \mathrm{Pe}$ & 0,987 \\
\hline
\end{tabular}


que foi de $18,2 \mathrm{~kg}$ de MS por $\mathrm{kg}$ de $\mathrm{P}$ (Quadro 5). O tratamento T5 $(350+70)$ concentra a localização do P na "cova" 10 vezes em relação ao T14 $(35+133)$ e diminui a dose no solo "externo". Para entender melhor essa localização, devem-se observar os efeitos dos tratamentos T3 $(525+35)$ e T15 $(350+70 \mathrm{~L})$.

$\mathrm{O}$ tratamento $\mathrm{T} 3$, em que se adicionaram unicamente $445,2 \mathrm{mg} /$ vaso de $\mathrm{P}$, apresentou produção de $23,71 \mathrm{~g} /$ vaso de MS de plantas de eucalipto aumento de $6308 \%$ em relação à resposta obtida no tratamento T14 (35 + 133). Também a eficiência do $\mathrm{P}$ adicionado foi bem maior: $53,2 \mathrm{~kg}$ de MS por $\mathrm{kg}$ de $\mathrm{P}$. $\mathrm{O}$ tratamento adicional T15 (350-70L), com doses semelhantes às do T5, mas sem incubação e com a dose de Pe aplicada de forma localizada (Figura 1b), apresentou produção de MS superior em relação ao T5 de 55,79\% e 42,70\%, para os solos LVAd-VI e LVAd-LS, respectivamente, em razão da falta de incubação e da localização da dose de Pe (Quadro 5). Esses resultados também foram encontrados por Leite et al. (2006) para a cultura do milho.

No solo LVAd-LS, a tendência foi a mesma em relação à produção de $\mathrm{MS}$, mas com maiores aumentos na eficiência do $\mathrm{P}$ adicionado (Quadro 5), por terem sido usadas menores doses de $\mathrm{P}$ e por apresentar este solo menor capacidade-tampão para $\mathrm{P}$.

Maior eficiência no aproveitamento do $\mathrm{P}$ adicionado, de maneira geral, foi obtida com a aplicação de maiores doses de P ao solo da "cova" (Quadro 5). Segundo Randall \& Hoeft (1988), em solos deficientes em P, a localização na zona radicular efetiva geralmente resulta em maior eficiência no uso desse macronutriente. Para Sleight et al. (1984), o uso mais eficiente do $\mathrm{P}$ pelas plantas jovens se dá quando o nutriente é colocado no solo, próximo à semente, onde há alto crescimento de raízes. Esse fato foi comprovado por Anghinoni \& Barber (1980), em milho, Borkert \& Barber (1985), em soja, e Yao \& Barber (1986), em trigo. Esses autores verificaram que a aplicação localizada de $\mathrm{P}$ próxima das raízes aumenta a sua absorção por estas.

$\mathrm{O}$ coeficiente de utilização médio do $\mathrm{P}$ adicionado para o solo LVAd-LS foi duas vezes superior ao do solo LVAd-VI, em decorrência da diferença de poder-tampão entre os dois solos. As produções foram semelhantes nos dois solos (Quadro 5). Por ser de textura média, o solo LVAd-LS deixa maior proporção do $\mathrm{P}$ adicionado disponível às plantas que o solo LVAd-VI, de textura muito argilosa (Quadro 3).

As superfícies de resposta mostram alta resposta do eucalipto às doses de $\mathrm{P}$ aplicadas à "cova" e que, para a amplitude utilizada, não foi alcançado um ponto de produção máxima (Quadro 6). Por outro lado, não houve resposta do eucalipto às doses de $\mathrm{P}$ do "solo externo" nem mesmo na presença de baixas doses aplicadas à "cova".

Esse comportamento do eucalipto confirma a alta exigência por $\mathrm{P}$ na fase inicial de crescimento da cultura (Novais et al., 1982, 1986). Como a muda foi transplantada com sistema radicular pequeno (devido

Quadro 5. Produção de matéria seca da parte aérea de plantas de eucalipto (MS), de acordo com as doses de $P$ aplicadas na "cova" (Pc) e no solo "externo" (Pe), quantidades totais aplicadas no vaso (Pav) e eficiência de utilização do $P$ adicionado nos solos LVAd-VI e LVAd-LS

\begin{tabular}{|c|c|c|c|c|c|c|c|}
\hline \multicolumn{2}{|c|}{ Tratamento } & \multicolumn{3}{|c|}{ LVAd-VI } & \multicolumn{3}{|c|}{ LVAd-LS } \\
\hline $\mathbf{N}^{o}$ & $\mathbf{C}+\mathbf{E}$ & P aplicado & Matéria seca & Efi.do Pad. ${ }^{(2)}$ & P aplicado & Matéria seca & Efi.do Pad. ${ }^{(2)}$ \\
\hline & & $\longrightarrow \mathrm{mg}$ & /vaso _ & $\mathrm{kg} \mathrm{kg}^{-1}$ & $-\mathrm{g}$ & raso & $\mathrm{kg} \mathrm{kg}^{-1}$ \\
\hline $\mathrm{T} 1$ & $-0,50:-0,50$ & 259,7 & 1,38 & 5,3 & 148,4 & 7,06 & 47,6 \\
\hline $\mathrm{T} 2$ & $-0,50: 0,50$ & 593,6 & 1,21 & 2,0 & 339,2 & 5,98 & 17,6 \\
\hline $\mathrm{T} 3$ & $0,50:-0,50$ & 445,2 & 23,71 & 53,2 & 254,4 & 17,21 & 67,6 \\
\hline $\mathrm{T} 4$ & $0,50: 0,50$ & 779,1 & 24,09 & 30,9 & 445,4 & 20,64 & 46,4 \\
\hline $\mathrm{T} 5$ & $0,00: 0,00$ & 519,4 & 9,43 & 18,2 & 296,8 & 10,44 & 35,2 \\
\hline $\mathrm{T} 6$ & $-0,75: 0,00$ & 380,3 & 0,21 & 0,5 & 217,3 & 1,54 & 7,1 \\
\hline $\mathrm{T} 7$ & $0,75: 0,00$ & 658,5 & 21,40 & 32,5 & 376,3 & 15,96 & 42,4 \\
\hline $\mathrm{T} 8$ & $0,00:-0,75$ & 269,0 & 14,51 & 54,0 & 153,7 & 12,61 & 82,1 \\
\hline $\mathrm{T} 9$ & $0,00: 0,75$ & 769,8 & 15,08 & 19,6 & 439,9 & 12,90 & 29,3 \\
\hline $\mathrm{T} 10$ & $-0,90:-0,50$ & 185,5 & 0,03 & 0,1 & 106,0 & 0,58 & 5,5 \\
\hline $\mathrm{T} 11$ & $-0,50:-0,90$ & 126,1 & 1,86 & 14,8 & 72,1 & 9,34 & 129,6 \\
\hline $\mathrm{T} 12$ & $0,90: 0,50$ & 853,3 & 19,51 & 22,9 & 487,6 & 18,40 & 37,7 \\
\hline $\mathrm{T} 13$ & $0,50: 0,90$ & 912,7 & 24,65 & 27,0 & 521,5 & 18,21 & 34,9 \\
\hline $\mathrm{T} 14$ & $-0,90: 0,90$ & 653,0 & 0,37 & 0,6 & 374,2 & 0,73 & 1,9 \\
\hline \multirow[t]{2}{*}{$\mathrm{T} 15$} & $0,00: 0,00 \mathrm{~L}^{(1)}$ & 519,4 & $21,33^{* *}$ & 41,0 & 296,8 & $18,22^{* *}$ & 61,4 \\
\hline & Média & 528,3 & 11,92 & 20,5 & 308,6 & 11,32 & 43,1 \\
\hline
\end{tabular}

(1) Tratamento adicional em que não houve incubação e a dose de Pe foi localizada. ${ }^{(2)}$ Produção de matéria seca de acordo com a quantidade de $\mathrm{P}$ aplicada. **: diferença significativa entre o tratamento 15 e seu equivalente (T5) no solo LVAd-VI e no solo LVAd-LS a $1 \%$. 
Quadro 6. Equações de regressão da produção de matéria seca da parte aérea (MS) de plantas de eucalipto (g/vaso), como variável dependente das doses de $\mathrm{P}\left(\mathrm{mg} \mathrm{dm}^{-3}\right)$, aplicadas na "cova" (Pc) e no "solo externo" (Pe), ou das quantidades totais de P, aplicadas no vaso (Pav, em mg/vaso), nos solos LVAd-VI e LVAd-LS

\begin{tabular}{cc}
\hline Equação & $\mathbf{R}^{2}$ \\
\hline \multicolumn{1}{c}{ Solo LVAd-VI } & 0,907 \\
$\hat{\mathrm{M}} \mathrm{S}=-3,175+0,05899^{* *} \mathrm{Pc}-0,10281^{*} \mathrm{Pe}-0,000022^{*} \mathrm{Pc}^{2}+0,000822^{* *} \mathrm{Pe}^{2}$ & 0,398 \\
$\hat{\mathrm{M}} \mathrm{S}=-8,752-1,0803000^{*} \mathrm{Pav}^{1 / 2}+0,0501428^{* *} \mathrm{Pav}$ & 0,962 \\
Solo LVAd-LS & 0,364 \\
$\hat{\mathrm{M}} \mathrm{S}=0,978+0,09225^{* *} \mathrm{Pc}-0,18127^{*} \mathrm{Pe}-0,000092^{* *} \mathrm{Pc}^{2}+0,002158^{* *} \mathrm{Pe}^{2}$ & \\
$\hat{\mathrm{M}} \mathrm{S}=8,836-0,0260838^{* *}$ Pav $+0,0000887^{* *} \mathrm{Pav}^{2}$ &
\end{tabular}

à poda) e virtualmente desprovida de reservas (germinação em areia lavada), passou a ser altamente dependente da disponibilidade inicial de $\mathrm{P}$ do solo ("cova"). Na presença de doses baixas na "cova" a muda não se desenvolveu e o incipiente sistema radicular não atingiu - e, assim, não explorou - o "solo externo". Por outro lado, na presença de doses altas na "cova", a planta cresceu e seu sistema radicular explorou todo o vaso, porém o Pc satisfez as exigências da planta. A resposta ao Pe é pequena e inconsistente (Quadro 6). Por sua vez, Leite et al. (2006) verificaram resposta do milho ao $\mathrm{P}$ do "solo externo" na presença de doses baixas na "cova" e a explicaram por uma maior reserva da semente, que proporcionou maior crescimento das raízes, de modo a atingir o "solo externo" e explorar o P dessa região. Portanto, a distância da faixa a ser fertilizada, no caso da localização do $\mathrm{P}$, deve levar em consideração a capacidade da cultura de possuir reservas suficientes para o crescimento da raiz até essa faixa.

\section{Doses recomendáveis de $P$}

Em razão de a resposta para $\mathrm{Pe}$ ter sido insignificante, adotou-se a menor dose do espaço experimental estudado como a recomendável para o "solo externo". Como não foram atingidas produções máximas no espaço fatorial, para a "cova" adotou-se a dose necessária para obtenção de $90 \%$ da maior produção, dentro da menor dose de Pe, como sendo a recomendável (Quadro 7).

Caso a quantidade de $\mathrm{P}$ recomendável para o vaso seja aplicada uniformemente ao volume de solo $\left(5,3 \mathrm{dm}^{3}\right)$ e não de forma localizada ("cova e "solo externo"), a produção estimada com as equações do quadro 6 seria de 0,31 g/vaso no solo LVAd-LS, enquanto no solo LVAd-VI seria negativa (-2,66 g/vaso) (Quadro 7). Portanto, com a mesma quantidade de $\mathrm{P}$ aplicada ao vaso, passar-se-ia de uma condição de inviabilidade de cultivo para outra de produção ideal (90 \% da produção máxima), simplesmente por localizar maior parte do P na "cova". Localizar o P aumenta sua eficiência de aproveitamento, por aumentar a chance do contato raiz: $\mathrm{P}$ e diminuir o tempo para que esse contato ocorra (Sleight et al., 1984), e há maior probabilidade de ocorrer maior disponibilidade de $\mathrm{P}$ na fase inicial do desenvolvimento da planta, na qual há alta demanda por $\mathrm{P}$, como verificado para trigo (Romer \& Schiling, 1986), milho (Anghinoni, 1992; Leite et al., 2006) e o próprio eucalipto (Neves, 1983).

$\mathrm{A}$ ausência de resposta às doses de $\mathrm{P}$ no "solo externo" na presença de altas doses de P na "cova" e a não obtenção de um máximo de produção no espaço experimental utilizado sugerem a necessidade do uso de doses ainda maiores para a "cova". Essa sugestão é reforçada pela resposta favorável à localização da dose de P do "solo externo" no tratamento adicional.

\section{Níveis críticos de fósforo na "cova" e no "solo externo"}

Foram calculados os níveis críticos de P na "cova" e no "solo externo" (Quadro 8) a partir das respectivas doses recomendáveis, substituindo-se os valores dessas doses nas equações para os teores de $\mathrm{P}$ recuperado do solo pelo extrator Mehlich-1, em função das doses aplicadas (Quadro 4).

Os elevados valores de níveis críticos na "cova" (Quadro 8), estão de acordo com os resultados obtidos em outros trabalhos com eucalipto, em casa de vegetação, com aplicação uniforme do $\mathrm{P}$ em todo o volume do solo (Novais et al., 1982; Neves, 1983). O nível crítico para o "solo externo" é muito inferior ao da "cova". Entretanto, como a fração de volume de solo a ser fertilizada é pequena, a quantidade a ser aplicada não foi grande. Novais et al. (1986) recomendam, na implantação, adubações com $\mathrm{P}$ na cova ou sulco de plantio para atingir os elevados valores de níveis críticos de $\mathrm{P}$, para a fase jovem do eucalipto. Já em fase mais adulta da planta (manutenção), o nível crítico de $\mathrm{P}$ é bem inferior e refere-se ao solo fora da cova ou sulco, que estará sendo explorado nessa fase da cultura. Eles encontraram níveis críticos de $\mathrm{P}$ nos solos arenoso e argiloso de 80 
Quadro 7. Doses recomendáveis de P para o solo da "cova" e solo "externo" para obtenção de 90 \% da maior produção de matéria seca (MS) da parte aérea de eucalipto, quantidade equivalente de $P$ a ser aplicada no vaso e eficiência de utilização do $P$ adicionado, nos solos LVAd-VI e LVAd-LS

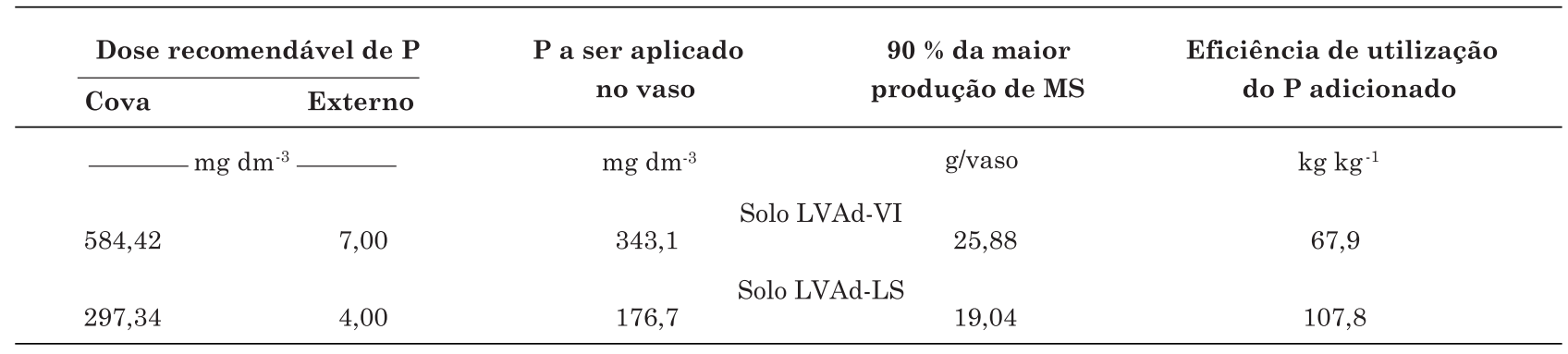

Quadro 8. Níveis críticos de P do solo da "cova" e do solo "externo", pelo extrator Mehlich-1, para a obtenção de 90 \% da maior produção da parte aérea de plantas de eucalipto, nos solos LVAdVI e LVAd-LS

\begin{tabular}{ccc}
\hline \multirow{2}{*}{ Solo } & \multicolumn{2}{c}{ Solo } \\
\cline { 2 - 3 } & Cova & "Externo" \\
\cline { 2 - 3 } & \multicolumn{2}{c}{$\mathrm{mg} \mathrm{dm}^{-3}-$} \\
LVAd-VI & 114,8 & 0,8 \\
LVAd-LS & 93,1 & 1,3 \\
\hline
\end{tabular}

e $60 \mathrm{mg} \mathrm{dm}^{-3}$, respectivamente, na fase inicial, reduzindo drasticamente na fase de manutenção $(4,5$ e $6,5 \mathrm{mg} \mathrm{dm}^{-3}$, respectivamente). Assim, estudos de fontes e localização da adubação fosfatada são necessários para se pensar na possibilidade de uma aplicação de uma fonte solúvel de P na "cova", a fim de suprir a necessidade da planta em sua fase inicial e de fosfato natural no solo "externo" para a fase adulta.

Na obtenção de altas produções de MS praticamente não houve resposta ao P do solo "externo"; adotou-se então, para critério de recomendação, a fixação de um valor de nível crítico somente para a "cova". Dessa forma, por simulação, para quaisquer teores do $\mathrm{P}$ original do solo, a recomendação de adubação a ser aplicada na "cova" será aquela que eleve o teor de $\mathrm{P}$, nessa região, para o valor do nível crítico (Quadro 9).

Estimou-se, então, a dose de $\mathrm{P}$ a ser recomendada (DR) para a "cova", para cada teor de P original do solo, empregando-se a fórmula:

$$
\mathrm{DR}=(\mathrm{NiCri}-\mathrm{Pd}) / \Delta(\mathrm{Prp} / \mathrm{Pad})
$$

em que NiCri é o nível crítico estimado para a "cova" (Quadro 8); Pd, o teor de P disponível em condições de solos de baixa, média e alta fertilidade, dentro do intervalo experimental de doses para o solo "externo"; e $\Delta(\operatorname{Prp} / \mathrm{Pad})$, a declividade obtida da equação de $\mathrm{P}$ disponível, em função da dose de $\mathrm{P}$ adicionada à "cova".
As doses a serem recomendadas e as quantidades de $\mathrm{P}$ a serem adicionadas diminuem à medida que cresce o teor de $\mathrm{P}$ do solo original, pois menor será a quantidade de P necessária para que se alcance o nível crítico (Quadro 9). Os mesmos resultados foram encontrados por Leite et al. (2006), trabalhando com milho em condições semelhantes às deste trabalho.

Os teores de $\mathrm{P}$ recuperado da mistura do solo da parte superior do vaso (Prs) e parte do solo "externo" mais a "cova" $(\mathrm{C}+\mathrm{E} 1$, Figura 1$)$ após a colheita do eucalipto foram relacionados com as doses de $\mathrm{P}$ aplicadas na "cova" e no solo "externo" (Pc e Pe) e com as quantidades de $\mathrm{P}$ aplicadas em todo o solo do vaso (Pav) ou no solo da parte superior deste (Pas) (Quadro 10).

Valores elevados de $\mathrm{R}^{2}$ obtidos para as equações do quadro 10 evidenciam que essa forma de amostragem foi representativa para a determinação do teor médio de $\mathrm{P}$ do volume de solo amostrado. A aplicação localizada dos fertilizantes nas linhas de semeadura restringe a aleatorização das amostras simples, pois aumenta a variabilidade horizontal dos teores dos nutrientes, principalmente para aqueles pouco móveis no solo e com maior efeito residual ( $\mathrm{P} \mathrm{e} \mathrm{K})$. Essa maior variabilidade foi encontrada por Salet et al. (1996) e Kray et al. (1998) em plantio direto. Na amostragem do solo para implantação do eucalipto em áreas que receberam adubação localizada de $\mathrm{P}$, recomenda-se a amostragem após o preparo mecânico do terreno, pois ocorre mistura dos teores de $\mathrm{P}$, uma vez que estes podem variar de acordo com as proporções de terra provenientes do sulco e das entrelinhas (Vasconcelos et al., 1982). Nessas condições, se a amostragem for feita após a colheita e antes do revolvimento do solo subsequente, a variabilidade dos índices de fertilidade do solo será semelhante àquela verificada no plantio direto e maior do que nas áreas sem cultivo (Alvarez V. \& Guarçoni M., 2003). Para contornar o problema da variabilidade dos teores dos nutrientes com a aplicação localizada dos fertilizantes, Alvarez V. \& Guarçoni M. (2003) recomendam que na amostragem do solo sob plantio direto e, ou, sob plantio convencional, após a colheita e antes do revolvimento 
Quadro 9. Doses a serem recomendadas e quantidades de $P$ a serem aplicadas na “cova” $\left(0,53 \mathrm{dm}^{3}\right) \mathrm{em} \mathrm{solos}^{\circ}$ com três diferentes teores de $P$ disponíveis para atingir o nível crítico de eucalipto, nos solos LVAd-VI e LVAd-LS

\begin{tabular}{|c|c|c|c|}
\hline Teor de P no solo & $\begin{array}{l}\text { Nível crítico de } \\
\text { P na "cova"(1) }\end{array}$ & $\begin{array}{l}\text { Dose de P a ser } \\
\text { recomendada na "cova" }\end{array}$ & $\begin{array}{c}\text { P a ser aplicado } \\
\text { na "cova" }\end{array}$ \\
\hline \multirow{2}{*}{\multicolumn{4}{|c|}{$\mathrm{mg} \mathrm{dm}^{-3}$}} \\
\hline & & & \\
\hline 0,8 & 115,0 & 582,1 & 308,5 \\
\hline 7,8 & 115,0 & 555,4 & 294,4 \\
\hline \multirow[t]{2}{*}{17,5} & 115,0 & 517,3 & 274,2 \\
\hline & & & \\
\hline 1,3 & 93,0 & 293,5 & 155,6 \\
\hline 9,4 & 93,0 & 271,0 & 143,6 \\
\hline 17,5 & 93,0 & 248,2 & 131,5 \\
\hline
\end{tabular}

(1) Níveis críticos definidos para atingir $90 \%$ da produção de matéria seca da parte aérea de plantas de eucalipto, obtida com a maior dose de P aplicada na "cova".

Quadro 10. Equações de regressão dos teores de $\mathbf{P}$ recuperados pelo extrator Mehlich-1 ( $\mathrm{mg} \mathrm{dm}^{-3}$ de P) da mistura do solo da parte superior do vaso (Prs), "cova" mais solo "externo" (C + E1), amostrada após o corte do eucalipto, como variável dependente das doses de $\mathrm{P}\left(\mathrm{mg} \mathrm{dm}^{-3}\right)$ aplicadas na "cova" (Pc) e no solo "externo" (Pe) e das quantidades de $\mathrm{P}$ aplicadas (mg) em todo o solo do vaso (Pav) e no solo da parte superior do vaso (Pas), nos solos LVAd-VI e LVAd-LS

\begin{tabular}{cc}
\hline Equação & $\mathbf{R}^{2}$ \\
\hline Solo LVAd-VI & \\
$\hat{\mathrm{P}}_{\mathrm{rs}}=-1,745+0,022401^{* *} \mathrm{Pc}+0,097029^{* *} \mathrm{Pe}$ & 0,987 \\
$\hat{\mathrm{P}}_{\mathrm{rs}}=1,434+0,013425^{* *} \mathrm{Pav}+0,000013^{* *} \mathrm{Pav}^{2}$ & 0,919 \\
$\hat{\mathrm{P}}_{\mathrm{rs}}=-1,814+0,038515^{* *} \mathrm{Pas}$ & 0,982 \\
$\quad$ Solo LVAd-LS & \\
$\hat{\mathrm{P}}_{\mathrm{rs}}=-0,872+0,034662^{* *} \mathrm{Pc}+0,177949^{* *} \mathrm{Pe}$ & 0,987 \\
$\hat{\mathrm{P}}_{\mathrm{rs}}=1,275+0,030042^{* *} \mathrm{Pav}+0,000025^{\circ} \mathrm{Pav}^{2}$ & 0,946 \\
$\hat{\mathrm{P}}_{\mathrm{rs}}=-0,878+0,064800^{* *} \mathrm{Pas}$ & 0,987 \\
\hline${ }^{\circ},{ }^{*},{ }^{* *}:$ significativos a 10,5 e $1 \%$, respectivamente.
\end{tabular}

Quadro 11. Equações de regressão da produção de matéria seca da parte aérea de plantas de eucalipto (MS, em g/vaso), como variável dependente do $P$ recuperado $\left(\mathrm{mg} \mathrm{dm}^{-3}\right)$ pelo extrator Mehlich-1 da mistura do solo $(\mathrm{C}+\mathrm{E} 1)$ da parte superior do vaso (Prs), amostrada após o corte do eucalipto, nos solos LVAd-VI e LVAd-LS

\begin{tabular}{ccc}
\hline Solo & Equação & $\mathbf{R}^{2}$ \\
\hline LVAd-VI & $\hat{\mathrm{M} S}=-3,828+1,17564^{* *} \operatorname{Prs}$ & 0,672 \\
LVAd-LS & $\hat{\mathrm{M} S}=12,960-7,29805^{* *} \operatorname{Prs}^{1 / 2}+1,77551^{* *} \operatorname{Prs}$ & 0,519 \\
\hline
\end{tabular}

** Significativo a $1 \%$. do solo, utilize-se trado de caneca na coleta de $17 \%$ das amostras simples no sulco de plantio, $33 \%$ a $10 \mathrm{~cm}$ do sulco e $50 \%$ no ponto médio entre sulcos.

$\mathrm{O}$ teores de $\mathrm{P}$ recuperados do solo apresentaram relação muito baixa com a produção de matéria seca do eucalipto, comparada àquela obtida em função das doses de Pc e Pe (solo LVAd-VI: $\mathrm{R}^{2}=0,907$ e solo LVAdLS: $R^{2}=0,962$ ) (Quadro 11). Esse fato deixa claro que os teores de $\mathrm{P}$ obtidos podem representar o volume do solo cultivado, mas não representam bem a disponibilidade efetiva para o crescimento inicial das plantas nesse volume de solo. Então, a forma de amostragem deve considerar, também, a exploração do volume de solo pela planta e sua resposta em termos de crescimento.

Substituindo as doses de Pc e Pe recomendáveis ou a quantidade equivalente de $\mathrm{P}$ aplicada à amostra do vaso (Pav) ou ao volume amostrado (Pas), nas respectivas equações para $\mathrm{P}$ recuperado do solo, após a colheita do eucalipto, foram obtidos os níveis críticos. Esses níveis críticos correspondem aos teores de P no solo relacionados com $90 \%$ da maior produção de MS (Quadro 12). Utilizar algum desses valores como um único nível crítico médio para o vaso não é confiável, pois o mesmo teor pode ser obtido a partir de diferentes combinações de doses de P na "cova" e no solo "externo".

\section{Simulação da amostragem do solo}

Segundo Alvarez V. et al. (1986), uma indagação que ocorre ao amostrar o solo, para fins de calibração, quando se localiza o $\mathrm{P}$, diz respeito à proporção de amostras a serem tomadas do sulco e das entrelinhas para compor uma amostra composta. Foram feitas simulações para amostragem com coletas de 20 ou de 21 amostras simples. Para isso, calculou-se, como único valor de $\mathrm{P}$ disponível para cada vaso, a média ponderada com o uso de diferente número de amostras 
simples e dos teores de P da "cova" e do solo "externo" (Pm) e ajustaram-se equações de regressão da produção de MS em função desses valores (Quadro 13).

Para ambos os solos, à medida que aumenta a proporção de amostras simples para a "cova", estreitase a relação com a produção de MS, de modo que a melhor relação é obtida com toda a ponderação dada à "cova" (20 amostras), corroborando os resultados

Quadro 12. Níveis críticos médios de $P$ no solo do vaso, após a colheita, para obtenção de $90 \%$ da maior produção da parte aérea de plantas de eucalipto, calculados a partir das equações ajustadas em função das doses de $P$ aplicadas na "cova" e no solo "externo" (Pc e Pe) e das quantidades de $P$ aplicadas em todo o solo do vaso (Pav) e na parte superior (Pas), nos solos LVAd-VI e LVAd-LS

\begin{tabular}{cccc}
\hline \multirow{2}{*}{ Solo } & \multicolumn{3}{c}{$\begin{array}{c}\text { Variáveis independentes das } \\
\text { equações utilizadas }\end{array}$} \\
\cline { 2 - 4 } & Pc e Pe & Pav & Pas \\
\hline & \multicolumn{4}{c}{$\mathrm{mg} \mathrm{dm}^{-3} \mathrm{de} \mathrm{P}$} & \\
LVAd-VI & 12,0 & 7,5 & 10,9 \\
LVAd-LS & 10,2 & 7,4 & 10,1 \\
\hline
\end{tabular}

encontrados por Leite et al. (2006), trabalhando com a cultura do milho em casa de vegetação. No entanto, o valor do $\mathrm{R}^{2}$ é pouco superior aos obtidos com a tomada de 10 amostras na "cova" e de 10 no solo "externo".

Mesmo observando valores semelhantes de $\mathrm{R}^{2}$ para as equações de produção de MS em função do teor médio de $\mathrm{P}$, ponderado pelo número de amostras simples da "cova" e do solo "externo", deve-se atentar para o fato de que o teor de Pm foi bem discrepante quando o número de amostras simples da "cova" e do solo "externo" foi de $20+0$ ou de $10+10$.

Essas variações indicam que os valores das declividades do teor de $\mathrm{P}$ recuperado, em função das doses de $\mathrm{P}$ adicionadas $(\Delta \mathrm{Prp} / \Delta \mathrm{Pad})$, aumentaram conforme se aumentou o numero de amostras simples da "cova". Esse aumento justifica as modificações dos valores dos níveis críticos dos quadros 8 e 12. Assim, em concordância com a proposição da utilização de um único valor de nível crítico, o da "cova", sugere-se que a amostragem para fins de calibração da adubação fosfatada, para o modo de localização utilizado, seja feita apenas na cova. Essa forma de amostragem significa que se passaria a dar maior ênfase à fertilidade do solo onde as mudas serão transplantadas e não à fertilidade média do terreno e, também, que dificilmente serão encontrados solos ricos em $\mathrm{P}$ que não precisem de adubação com fosfatos solúveis na implantação de lavouras de eucalipto.

Quadro 13. Equações de regressão da produção de matéria seca (MS) da parte aérea de plantas de eucalipto (g/vaso), como variável dependente da média ponderada (Pm), pelo número de amostras simples, dos teores de $\mathrm{P}\left(\mathrm{mg} \mathrm{dm}^{-3}\right.$ de P) recuperados da "cova" (Prc) e do solo"externo" (Pre), nos solos LVAd-VI e LVAd-LS

\begin{tabular}{|c|c|c|c|}
\hline \multicolumn{2}{|c|}{ Número ${ }^{(1)}$} & \multirow{2}{*}{ Equação } & \multirow{2}{*}{$\mathbf{R}^{2}$} \\
\hline Prc & Pre & & \\
\hline \multirow{2}{*}{0} & \multicolumn{3}{|c|}{ Solo LVAd-VI } \\
\hline & 20 & $\hat{\mathrm{M}} \mathrm{S}=7,569+1,43892^{* *} \mathrm{Pm}-0,05523^{* *} \mathrm{Pm}^{2}$ & 0,076 \\
\hline 1 & 20 & $\hat{\mathrm{M} S}=1,897+0,82345^{* *} \mathrm{Pm}$ & 0,269 \\
\hline 7 & 14 & $\hat{\mathrm{M}} \mathrm{S}=-7,635+1,04980^{* *} \mathrm{Pm}-0,00875^{* *} \mathrm{Pm}^{2}$ & 0,867 \\
\hline & 10 & $\hat{\mathrm{M}} \mathrm{S}=-6,832+0,79969^{* *} \mathrm{Pm}-0,00538^{* *} \mathrm{Pm}^{2}$ & 0,908 \\
\hline 20 & 1 & $\hat{\mathrm{M}} \mathrm{S}=-4,093+0,40692^{* *} \mathrm{Pm}-0,00153^{* *} \mathrm{Pm}^{2}$ & 0,911 \\
\hline 20 & 0 & $\hat{\mathrm{M}} \mathrm{S}=-3,907+0,38498^{* *} \mathrm{Pm}-0,00138^{* *} \mathrm{Pm}^{2}$ & 0,910 \\
\hline 0 & 20 & $\hat{\mathrm{M}} \mathrm{S}=\quad \begin{array}{r}\text { Solo LVAd-LS } \\
8,375+0,24662^{* *} \mathrm{Pm}\end{array}$ & 0,040 \\
\hline 1 & 20 & $\hat{\mathrm{M}} \mathrm{S}=7,787-0,21202 \quad \mathrm{Pm}+0,03063^{* *} \mathrm{Pm}^{2}$ & 0,249 \\
\hline 7 & 14 & $\hat{\mathrm{M} S}=-2,074+0,67730^{* *} \mathrm{Pm}-0,00510^{* *} \mathrm{Pm}^{2}$ & 0,834 \\
\hline 10 & 10 & $\hat{\mathrm{M}} \mathrm{S}=1,963+0,55844^{* *} \mathrm{Pm}-0,00373^{* *} \mathrm{Pm}^{2}$ & 0,900 \\
\hline 20 & 1 & $\hat{\mathrm{M} S}=-0,364+0,30952^{* *} \mathrm{Pm}-0,00127^{* *} \mathrm{Pm}^{2}$ & 0,937 \\
\hline 20 & 0 & $\hat{\mathrm{M}} \mathrm{S}=-0,243+0,29424^{* *} \mathrm{Pm}-0,00116^{* *} \mathrm{Pm}^{2}$ & 0,938 \\
\hline
\end{tabular}

(1) Número de vezes em que o P da "cova" ou solo "externo" é considerado no cálculo do teor médio ponderado de P (Pm). *, **: significativos a 5 e $1 \%$, respectivamente. 


\section{CONCLUSÕES}

1. A localização da maior quantidade de $\mathrm{P}$ aplicada no solo da "cova" aumenta a eficiência de utilização do $\mathrm{P}$ adicionado.

2. A produção de matéria seca da parte aérea de plantas de eucalipto apresenta relação muito baixa com a quantidade de $\mathrm{P}$ aplicada por vaso (Pav), mas depende estreitamente das doses aplicadas à "cova" (Pc) e ao "solo externo" (Pe), mostrando o efeito da localização do P.

3. As superfícies de resposta $(\mathrm{MS}=\mathrm{f}(\mathrm{Pc}, \mathrm{Pe}))$ mostram que o crescimento inicial das plantas depende, essencialmente, das doses de $\mathrm{P}$ adicionadas na "cova".

4. Os valores dos níveis críticos para a "cova" são muito elevados. Por outro lado, para o "solo externo" são baixos. Como a fração do volume da "cova" é pequena (1/10), a quantidade recomendável de $\mathrm{P}$ para o vaso não é muito elevada.

5. O valor de $\mathrm{P}$ disponível na amostra de solo da parte superior retirada após a colheita $\left(\mathrm{C}+\mathrm{E}_{1}\right)$ representou bem a disponibilidade média do volume de solo amostrado em função do P adicionado na "cova" e na "parte externa". Contudo, não se relacionou bem com a produção de matéria seca das plantas de eucalipto.

6. Os níveis críticos médios de $\mathrm{P}$ no solo do vaso, após a colheita, são valores baixos e compatíveis com os obtidos usualmente em campo.

7. A estreita relação entre a produção de matéria seca e o P recuperado da "cova" evidencia o uso de um valor de nível crítico para esse local. Assim, na fase de calibração, bastaria apenas a coleta de amostras na "cova" (sulco).

\section{LITERATURA CITADA}

ALVAREZ V., V.H. Correlação e calibração de métodos de análise de solos. In: ALVAREZ V., V.H.; FONTES, L.E.F. \& FONTES, M.P.F., eds. O solo nos grandes domínios morfoclimáticos do Brasil e o desenvolvimento sustentado. Viçosa, MG, UFV/DPS, SBCS, 1996. p.615-646.

ALVAREZ V., V.H.; CAJUSTE, L.J. \& CRUZ DIAZ, J. Estudio de la variabilidad en el contenido de fósforo del suelo en función de las dosis y ubicación del fertilizante fosfatado en un experimento de maiz (Zea mays L.). Agrociencia, 64:23-32, 1986

ALVAREZ V., V.H. \& FONSECA, D.M. Definição de doses de fósforo para determinação da capacidade máxima de adsorção de fosfatos e para ensaios em casa-de-vegetação. R. Bras. Ci. Solo, 14:49-55, 1990.

ALVAREZ V., V.H. \& GUARÇONI M., A. Variabilidade horizontal da fertilidade do solo de uma unidade de amostragem em sistema plantio direto. R. Bras. Ci. Solo, $27: 297-310,2003$.
ALVAREZ V., V.H.; NOVAIS, R.F.; DIAS, L.E. \& OLIVEIRA, J.A. Determinação e uso do fósforo remanescente. B. Inf. SBCS, 52:27-32, 2000.

ANGHINONI, I. Uso de fósforo pelo milho afetado pela fração de solo fertilizada com fosfato solúvel. R. Bras. Ci. Solo, 16:349-353, 1992.

ANGHINONI, I. \& BARBER, S.A. Phosphorus application rate and distribution in the soil and phosphorus uptake by corn. Soil Sci. Soc. Am. J., 44:1041-1044, 1980.

BORKERT, C.M. \& BARBER, S.A. Soybean shoot and root growth and phosphorus concentration as affected by phosphorus placement. Soil Sci. Soc. Am. J., 49:152-155, 1985.

BRAY, R.H. A nutrient mobility concept of soil plant relationships. Soil Sci., 78:9-22, 1954.

GUARÇONI M., A. Efeito da localização de adubos fosfatados sobre o crescimento de plantas de milho. Viçosa, MG, Universidade Federal de Viçosa, 2001. 52p. (Tese de Mestrado)

HOLFORD, I.C.R. Soil phosphorus: Its measurement, and its uptake by plants. Aust. J. Soil Res., 35:227-239, 1997.

KRAY, C.H.; SALET, R.L. \& ANGHINONI, I. Variabilidade horizontal e amostragem do solo dirigida no sistema plantio direto. Porto Alegre, Universidade Federal do Rio Grande do Sul, 1998. 6p. (Relatório de pesquisa)

LEITE, P.B.; ALVAREZ V., V.H.; BARROS, N.F.; NEVES, J.C.L. \& GUARCONI M., A. Níveis críticos de fósforo, para milho, em casa de vegetação, de acordo com a sua localização no solo. R. Bras. Ci. Solo, 30:497-508, 2006.

LEITE, R.A. Uso de matrizes experimentais e de modelos estatísticos no estudo do equilíbrio fósforo-enxofre na cultura de soja em amostras de dois Latossolos de Minas Gerais. Viçosa, MG, Universidade Federal de Viçosa, 1984. 87p. (Tese de Mestrado)

MODEL, N.S. \& ANGHINONI, I. Resposta do milho a modos de aplicação de adubos e técnicas de preparo do solo. $\mathrm{R}$. Bras. Ci. Solo, 16:55-59, 1992.

NEVES, J.C.L. Aspectos nutricionais em mudas de Eucalyptus spp. - Tolerância ao alumínio e níveis críticos de fósforo no solo. Viçosa, MG, Universidade Federal de Viçosa, 1983. 87p. (Tese de Mestrado)

NEVES, J.C.L.; NOVAIS, R.F.; BARROS, N.F. \& MUNIZ, A.S. Níveis críticos de fósforo em diferentes solos e extratores químicos para o crescimento de mudas de eucalipto. Acta For. Bras., 2:63-79, 1987.

NOVAIS, R.F.; BARROS, N.F.; NEVES, J.C.L. \& COUTO, C. Níveis críticos de fósforo no solo para o eucalipto. $R$. Árvore, 6:29-37, 1982.

NOVAIS, R.F.; BARROS, N.F.; NEVES, J.C.L. \& COUTO, C. Interpretação de análise química do solo para o crescimento e desenvolvimento de Eucalyptus spp.: Níveis críticos de implantação e manutenção. R. Árvore, 10:105111,1986

NOVAIS, R.F. \& SMYTH, T.J. Fósforo em solo e planta em condições tropicais. Viçosa, MG, Universidade Federal de Viçosa, 1999. 399p. 
RANDALL, G.W. \& HOEFT, R.G. Placement methods for improved efficiency of $\mathrm{P}$ and $\mathrm{K}$ fertilizers: A review. J. Prod. Agric., 1:70-79, 1988.

ROMER, W. \& SCHILLING, G. Phosphorus requeriments of the wheat plant in various stages of its life cycle. Plant Soil, 91:221-229, 1986.

SALET, R.L.; KRAY, C.H.; FORNARI, T.G.; CONTE, E.; KOCHHANN, R.A. \& ANGHINONI, I. Variabilidade horizontal e amostragem de solo no sistema plantio direto. In: REUNIÃO SUL-BRASILEIRA DE CIÊNCIA DO SOLO, 1., Lages, 1996. Resumos Expandidos. Lages, Sociedade Brasileira de Ciência do Solo, 1996. p.74-76.
SLEIGHT, D.M.; SANDER, D.M. \& PETERSON, S.A. Effect of fertilizer phosphorus placement on the availability of phosphorus. Soil Sci. Soc. Am. J., 48:336-340, 1984.

VASCONCELOS, C.A.; SANTOS, H.L.; BAHIA FILHO, A.F.C.; OLIVEIRA, A.C. \& PACHECO, E.B. Amostragem de solo em área com adubação fosfatada aplicada a lanço e no sulco de plantio. R. Bras. Ci. Solo, 6:221-225, 1982.

YAO, J. \& BARBER, S.A. Effect on phosphorus rate placed in different soil volumes on $\mathrm{P}$ uptake and growth of wheat. Soil Sci. Soc. Am. J.,17:819-829, 1986. 